\title{
Retrospective analysis of non-laboratory-based adverse drug reactions induced by intravenous radiocontrast agents in a Joint Commission
} International-accredited academic medical center hospital in China

This article was published in the following Dove Press journal:

Therapeutics and Clinical Risk Management

26 April 2017

Number of times this article has been viewed

\author{
Qin-lan Chen' \\ Xiao-ying Zhao ${ }^{2}$ \\ Xiao-mi Wang' \\ $\mathrm{Na} \mathrm{Lv}^{2}$ \\ Ling-ling $\mathrm{Zhu}^{3}$ \\ Hui-min $\mathrm{Xu}^{4}$ \\ Quan Zhou ${ }^{4}$ \\ 'Radiology Nursing Unit, Division \\ of Nursing, ${ }^{2}$ Department of \\ Quality Management, ${ }^{3}$ Geriatric VIP \\ Care Ward, Division of Nursing, \\ ${ }^{4}$ Department of Pharmacy, The \\ Second Affiliated Hospital, School \\ of Medicine, Zhejiang University, \\ Hangzhou, Zhejiang, People's \\ Republic of China
}

Correspondence: Quan Zhou Department of Pharmacy, The Second Affiliated Hospital, School of Medicine, Zhejiang University, Jiefang Road No 88, Shangcheng District, Hangzhou 310009 , Zhejiang Province, People's Republic of China

Tel +86 57I 877846 I5

Fax +86 57| 87022776

Email zhouquan I42602@zju.edu.cn

Xiao-ying Zhao

Department of Quality Management, The Second Affiliated Hospital, School of Medicine, Zhejiang University, Jiefang Road No 88, Shangcheng District, Hangzhou 310009 , Zhejiang Province, People's Republic of China

Email zrxz@zju.edu.cn

\begin{abstract}
The authors retrospectively analyzed the pattern and characteristics of non-laboratorybased adverse drug reactions (ADRs) induced by intravenous radiocontrast agents in a large-scale hospital in China during 2014-2015. There were 314 ADR cases among 118,208 patients receiving enhanced CT or MRI examinations. The frequency of moderate/severe ADRs defined by Chinese Society of Radiology (ie, severe vomiting, systematic urticaria, facial swelling, dyspnea, vasovagal reaction, laryngeal edema, seizure, trembling, convulsions, unconsciousness, shock, death, and other unexpected adverse reactions) was rare $(0.0431 \%)$, whereas the mild ADRs were uncommon $(0.2225 \%)$ and accounted for $83.76 \%$ of ADRs. Frequency of ADRs induced by iodinated contrast agents was related with examination site, sex, and type of patient settings $(P<0.01)$ and was higher compared with gadolinium contrast agents $(0.3676 \%$ vs $0.0504 \%, P<0.01)$. From 2014 to 2015 , frequencies of total and moderate/severe ADRs induced by iodinated contrast agents decreased significantly $(0.4410 \%$ vs $0.2947 \%, P<0.01 ; 0.0960 \%$ vs $0.0282 \%, P<0.01$, respectively). Frequency of ADRs differed among different iodinated contrast and gadolinium contrast $(P<0.05)$ agents. Iopromide's ADR frequency in 2014 was significantly higher compared with iopamidol, ioversol, or iohexol $(P<0.01)$. Frequency of moderate/severe ADRs induced by iodixanol was 4.1-5.4 times that of iohexol, iopromide, or iopamidol. Rash was the predominant ADR subtype $(84.39 \%)$ and occurred more frequently with iodixanol compared with iohexol, iopamidol, or ioversol $(P<0.01)$. Overall, $21.97 \%$ of ADR cases had allergy history or atopy traits, and these cases experienced ADRs earlier than the negative ones ( $17.19 \mathrm{~min}$ vs $85.34 \mathrm{~min}, P<0.01)$. The mean time to onset of ADRs was increased in patients receiving iodixanol compared with other iodinated contrast agents (323.77 min vs $42.36 \mathrm{~min}, P<0.01$ ). Overall, $37.26 \%$ of ADRs occurred within 5 min and $84.08 \%$ of ADRs occurred within $30 \mathrm{~min}$. Efficient quality improvement in decreasing ADRs induced by radiocontrast agents has been achieved by multidisciplinary collaboration.
\end{abstract}

Keywords: allergy, atopy, contrast media, drug monitoring, gadolinium contrast, iodinated contrast, quality improvements

\section{Introduction}

Radiocontrast agents are used widely to improve medical imaging. Institute for Safe Medication Practices lists intravenous radiocontrast agents as high alert medications, which bear a heightened risk of causing significant harm to the patient when used in error. ${ }^{1}$ It is clinically important to ensure a proper monitoring of adverse drug reactions 
(ADRs) induced by radiocontrast agents during and after the procedure. But totally non-ionic iodinated contrast and gadolinium-based contrast agents are highly safe, and severe adverse events seldom occur under appropriate care. ${ }^{2,3}$

There is little literature on quality improvements in decreasing ADRs induced by radiocontrast agents. Brown et al reported that a 6-year regional multicenter quality improvement intervention could reduce contrast-induced acute kidney injury after percutaneous coronary interventions. ${ }^{4}$ Dykes et al reported that a national data registry and practice quality improvement initiative failed to have a statistically significant positive impact on intravenous contrast extravasation during $\mathrm{CT} .^{5}$ The aim of this study was to analyze the nature and incidence of non-laboratory-based ADRs induced by intravenous radiocontrast agents in a Joint Commission International (JCI)-accredited academic medical center hospital in China during a 2-year period, and investigate whether quality improvements in this respect could be achieved through multidisciplinary team's integrated endeavors.

\section{Methods}

\section{Data collection}

A retrospective analysis of ADRs induced by radiocontrast agents was performed in the Second Affiliated Hospital of Zhejiang University (SAHZU), a 3200-bed JCI-accredited academic medical center hospital with 3.5 million outpatient visits and 110,000 discharged patients annually (data in 2016) in Zhejiang Province, which has a population of $\sim 54.4$ million. The ADRs induced by radiocontrast agents during 2014-2015 were derived from a mandatory reporting system consisting of detailed written sheets in radiology nursing care unit and brief electronic online reporting. Data mining was performed, focusing on reporter's diagnosis of subtypes of ADRs, severity rating, injection time, time to onset of ADRs, demographic information (sex, age, body weight, whether patients had allergy history or atopy traits), kind of radiocontrast agent used, examination site, ADR treatment and outcomes.

According to guideline for iodinated contrast agents use published by Chinese Society of Radiology (CSR), ADRs induced by iodinated contrast agents could be divided into three categories, ${ }^{6}$ that is, mild ADRs (eg, cough, sneezing, transient chest tightness, conjunctivitis, rhinitis, nausea, systematic fever, urticaria, itching, and angioneurotic edema), moderate ADRs (eg, severe vomiting, systematic urticaria, facial swelling, dyspnea, and vasovagal reaction), and severe ADRs (eg, laryngeal edema, seizure, trembling, convulsions, unconsciousness, shock, death, and other unexpected adverse reactions). ADRs induced by gadolinium contrast agents were classified by referencing the method for iodinated contrast agents.

In this present study, we also classified several symptoms not included in the CSR criteria into three types, that is, mild or localized facial swelling, mild trembling or shivering, nasal congestion, single symptom such as mild gastrointestinal discomfort, feeling of binaural blockage, transient blurred vision, dizziness, and numb limbs as mild ADRs; substantial facial swelling, single systematic trembling or shivering, hypertention, chest distress, and palpitation as moderate ADRs; and single trembling or shivering coupled with severe systematic symptoms, oxygen desaturation as severe ADRs. Classification criteria of contrast media-related ADRs were consistent through the whole study period.

Drug and Therapeutics Committee (DTC) and the Ethics Committee of SAHZU approved this study. The Ethics Committee at SAHZU deemed that this program was in compliance with the Declaration of Helsinki and patient consent was unnecessary as this study was a review of previous data and did not involve patient data confidentiality. The data presented in the study are available in the archives of DTC of SAHZU. Access and use of these data need permission from the SAHZU DTC.

\section{Statistical analysis}

A descriptive analysis was performed. Chi-square tests were used for testing differences in occurrence rates between two groups using SPSS software (version 13.0, SPSS Incorporated, Chicago, IL, USA). Fisher's exact test was used when 2 cells $(50.0 \%)$ of a contingency table had expected count $<5$. Pearson Chi-square continuity correction was used when 1 cell $(25.0 \%)$ had expected count $<5$. Pearson chi-square was used when 0 cell had expected count $<5$. Student's $t$-test was used for testing difference between sample means. A $P$-value $<0.05$ was considered to be statistically significant. A $P$-value $<0.01$ was considered to be highly significant.

\section{Results}

\section{Demographic and clinical information}

Three hundred and fourteen ADR cases induced by radiocontrast agents were identified among 118,208 patients enrolled in various types of enhanced CT or MRI examinations during 2014-2015. Using the Naranjo probability scale, ${ }^{7}$ all reactions were "probable". Frequencies of mild and moderate/ severe ADRs induced by radiocontrast agents were $0.2225 \%$ $(263 / 118,208)$ and $0.0431 \%(51 / 118,208)$, respectively. 
There was no fatality induced by radiocontrast agents during the study period. The basic principles for the treatments of contrast media-related ADRs followed the guideline published by CSR. ${ }^{6}$ Adverse events required symptomatic and/or aggressive treatments in 268 cases $(85.35 \%)$, while 46 cases $(14.65 \%)$ were not specifically treated but remitted spontaneously. There were 11 patients transferred to emergency department for further observation. Four intrainsitutional first-aid calls were performed at radiology department and the average response time was $2.6 \mathrm{~min}$. Percentage of ADR recovery was $100 \%$.

Oral hydration was administered first for the cases with mild ADRs, whereas oral and intravenous hydration were given to the patients with moderate/severe ADRs. There were 243 ADR cases (77.39\%) receiving oral hydration and 28 cases $(8.92 \%)$ receiving sodium lactate ringer's infusion. Two hundred and sixty-two (83.44\%) ADR cases received parenteral corticosteroids, including intravenous dexamethasone $5 \mathrm{mg}$ for 4 cases and $10 \mathrm{mg}$ for 252 cases, intravenous methylprednisolone $40 \mathrm{mg}$ for 3 cases, and intramuscular injection of betamethasone/betamethasone dipropionate
( $2 \mathrm{mg} / 5 \mathrm{mg}$ ) for 2 cases. Topical corticosteroid ointment was given to 2 ADR cases. Five ADR cases (1.59\%) received intramuscular injection of $\mathrm{H} 1$ receptor blockers (ie, promethazine $12.5-25 \mathrm{mg}$ for 3 cases and chlorpheniramine $10 \mathrm{mg}$ for 2 cases), whereas 10 ADR cases received oral $\mathrm{H} 1$ receptor blockers such as loratadine and cetirizine. Electrocardiogram monitor was applied for 70 ADR cases (22.29\%). Oxygen inhalation was performed for 31 ADR cases (9.87\%). Epinephrine 0.5-1 mg was applied for 5 cases with severe ADRs. Sublingual nitroglycerin tablets were given to 2 moderate ADR cases. Fifty percent glucose solution $(20-100 \mathrm{~mL})$ was orally administrated for 1 ADR case and calcium gluconate $1 \mathrm{~g}$ was intravenously injected for 2 cases with urticaria.

Demographic and clinical information were listed in Table 1 . There was statistically significant sex difference in frequency of ADRs induced by $\mathrm{CT}$ radiocontrast agents in $2014(0.5792 \%$ vs $0.3459 \%, P<0.01)$ and during $2014-2015$ ( $0.4628 \%$ vs $0.3005 \%, P<0.01)$. Female patients were more susceptible to ADRs induced by iodinated contrast agents compared with male patients. Frequency of ADR in outpatients and emergency patients receiving iodinated

Table I Demographic and clinical information

\begin{tabular}{|c|c|c|c|}
\hline Data & 2014 & 2015 & 2014 and 2015 \\
\hline Relative percentage of male ADR cases to all ADR cases (\%) & $46.11 \%(83 / 180)$ & $48.51 \%(65 / 134)$ & $47.13 \%(|48 / 3| 4)$ \\
\hline Age of ADR cases (year), mean \pm SD & $51.6 \pm 15.0$ & $51.13 \pm 14.42$ & $51.42 \pm 14.70$ \\
\hline Body weight of ADR cases $(\mathrm{kg})$, mean $\pm \mathrm{SD}$ & $51.13 \pm 14.42$ & $61.36 \pm 11.29$ & $60.38 \pm 11.00$ \\
\hline Relative percentage of inpatients among all ADR cases & $36.11 \%(65 / 180)$ & $43.28 \%(58 / 134)$ & $39.17 \%(123 / 314)$ \\
\hline Relative percentage of ADR patients with allergy history or atopy & $23.33 \%(42 / 180)$ & $20.15 \%(27 / 134)$ & $21.97 \%(69 / 314)$ \\
\hline Percentage of drug intervention & $82.22 \%(\mid 48 / 180)$ & $89.55 \%(120 / 134)$ & $85.35 \%(268 / 3 \mid 4)$ \\
\hline Percentage of ADR recovery & $100 \%$ & $100 \%$ & $100 \%$ \\
\hline Cases of patient complaints & 2 & 0 & 2 \\
\hline Percentage of morning administration & $46.11 \%(83 / 180)$ & $40.30 \%(54 / 134)$ & $43.63 \%(|37 / 3| 4)$ \\
\hline \multicolumn{4}{|l|}{ Frequency of ADR in patients receiving iodinated contrast } \\
\hline Male & $0.3459 \%(79 / 22,84 I)$ & $0.2549 \%(58 / 22,750)$ & $0.3005 \%(137 / 45,591)$ \\
\hline Female & $0.5792 \%(91 / 15,712)^{\mathrm{a}, \mathrm{c}}$ & $0.3504 \%(57 / 16,269)$ & $0.4628 \%(|48 / 31,98|)^{a}$ \\
\hline \multicolumn{4}{|l|}{ Frequency of ADR in patients receiving iodinated contrast } \\
\hline Inpatients & $0.3034 \%(60 / 19,775)$ & $0.2608 \%(52 / 19,938)$ & $0.2820 \%(112 / 39,713)$ \\
\hline Outpatients and emergency patients & $0.5858 \%(110 / 18,778)^{b, c}$ & $0.3302 \%(63 / 19,081)$ & $0.4570 \%(173 / 37,859)^{b}$ \\
\hline \multicolumn{4}{|l|}{ Frequency of ADR in patients receiving gadolinium contrast } \\
\hline Male & $0.03797 \%(4 / 10,535)$ & $0.06282 \%(7 / I I, \mid 43)$ & $0.05074 \%(I I / 2 I, 678)$ \\
\hline Female & $0.06445 \%(6 / 9,309)$ & $0.1244 \%(12 / 9,649)$ & $0.09495 \%(18 / 18,958)$ \\
\hline \multicolumn{4}{|l|}{ Frequency of ADR in patients receiving gadolinium contrast } \\
\hline Inpatients & $0.05155 \%(5 / 9,699)$ & $0.05549 \%(6 / 10,812)$ & $0.05363 \%(I I / 20,51 I)$ \\
\hline Outpatients and emergency patients & $0.04929 \%(5 / 10,145)$ & $0.1303 \%(13 / 9,980)$ & $0.08944 \%(18 / 20,125)$ \\
\hline \multicolumn{4}{|c|}{ Frequency of $A D R$ induced by iodinated contrast based on examination site } \\
\hline Limbs & $1.1820 \%(5 / 423)^{d}$ & $0.5952 \%(2 / 336)$ & $0.9222 \%(7 / 759)^{d}$ \\
\hline Abdomen and pelvic & $0.5298 \%(85 / 16,043)^{c, d}$ & $0.1515 \%(25 / 16,497)$ & $0.3380 \%(110 / 32,540)^{d}$ \\
\hline Neck & $0.4548 \%(15 / 3,298)^{d}$ & $0.2237 \%(8 / 3,576)$ & $0.3346 \%(23 / 6,874)^{d}$ \\
\hline Head and brain & $0.3641 \%(13 / 3,570)^{d}$ & $0.2421 \%(9 / 3,717)$ & $0.3019 \%(22 / 7,287)^{d}$ \\
\hline Chest & $0.2899 \%(44 / 15,178)^{c, d}$ & $0.1083 \%(16 / 14,773)$ & $0.2003 \%(60 / 29,951)^{d}$ \\
\hline
\end{tabular}

Notes: ${ }^{a} P<0.01$ (female vs male); ${ }^{b} P<0.01$ (inpatients vs outpatients and emergency patients); ${ }^{c} P<0.01$ (20I4 vs 2015$)$; ${ }^{d} P<0.01$ (comparison in frequency of $A D R$ regarding different examination sites).

Abbreviations: ADR, adverse drug reaction; SD, standard deviation. 
contrast agents was higher compared with inpatients in 2014 $(0.5858 \%$ vs $0.3034 \%, P<0.01)$ and during $2014-2015$ $(0.4570 \%$ vs $0.2820 \%, P<0.01)$. However, the influence of sex or patient setting type was not observed in patients receiving gadolinium contrast agents.

From 2014 to 2015, significant reduction in frequency of ADRs induced by iodinated contrast agents was observed in female patients $(0.5792 \%$ vs $0.3504 \%, P<0.01)$, outpatients and emergency patients $(0.5858 \%$ vs $0.3302 \%, P<0.01)$, patients receiving abdominal and pelvic examination $(0.5298 \%$ vs $0.1515 \%, P<0.01)$, and chest examination $(0.2899 \%$ vs $0.1083 \%, P<0.01)$. There was statistically significant difference in frequency of ADRs induced by iodinated contrast agents based on examination sites (limbs $>$ abdomen and pelvic, neck, head and brain $>$ chest, $P<0.01)$.

\section{Frequency of ADRs induced by 12 kinds of radiocontrast agents}

Table 2 lists frequencies of ADRs induced by 12 kinds of radiocontrast agents during 2014-2015. Iodinated contrast agents exhibited a higher frequency of ADRs compared with gadolinium contrast agents $(0.3674 \%$ vs $0.0504 \%, P<0.01)$. From 2014 to 2015, the total frequency of ADRs caused by iodinated contrast agents significantly decreased $(0.4410 \%$ vs $0.2947 \%, P<0.01)$. With respect to moderate/severe ADRs, the frequency also reduced $(0.0960 \%$ vs $0.0282 \%, P<0.01)$. Statistically significant difference in frequencies of ADRs was observed with seven iodinated contrast agents in 2014 $(P<0.01)$, six iodinated contrast agents in $2015(P<0.01)$, and three gadolinium contrast agents in $2014(P<0.05)$. There was no significant difference in frequencies of ADRs induced by 2 products of iohexol $\left(\mathrm{Ousu}^{\circledR}\right.$ [Yangtze River Pharmaceutical Group, Taizhou, Jiangsu, People's Republic of China] vs Omnipaque ${ }^{\circledR}$ [GE Healthcare Shanghai Pharmaceutical Manufacturing Plant, Pudong, Shanghai, People's Republic of China]); however, significant difference in frequencies of ADRs was observed among patients receiving 2 products of gadopentetate dimeglumine in $2014(0.2670 \%$ vs $0.0218 \%, P<0.05)$. Frequency of ADRs induced by iopromide $(0.8115 \%)$ and iodixanol $(0.6957 \%)$ was higher compared with iopamidol $(0.2639 \%)$, ioversol $(0.1976 \%)$, or iohexol $(0.3072 \% ; P<0.01)$. Regarding moderate/severe ADRs, iodixanol had a higher frequency compared with iopromide, iopamidol, or iohexol $(P<0.01)$.

\section{Subtypes of ADRs}

Table 3 lists subtypes of ADRs induced by radiocontrast agents during 2014-2015. Rash was the predominant ADR

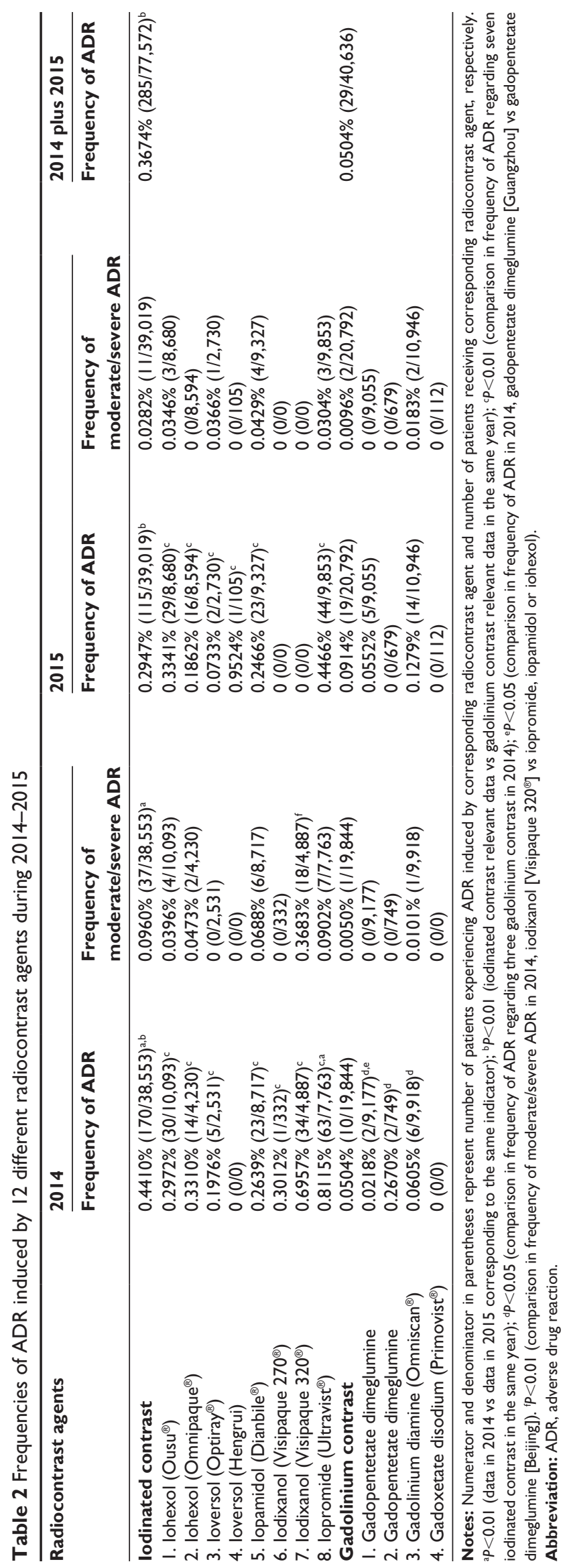




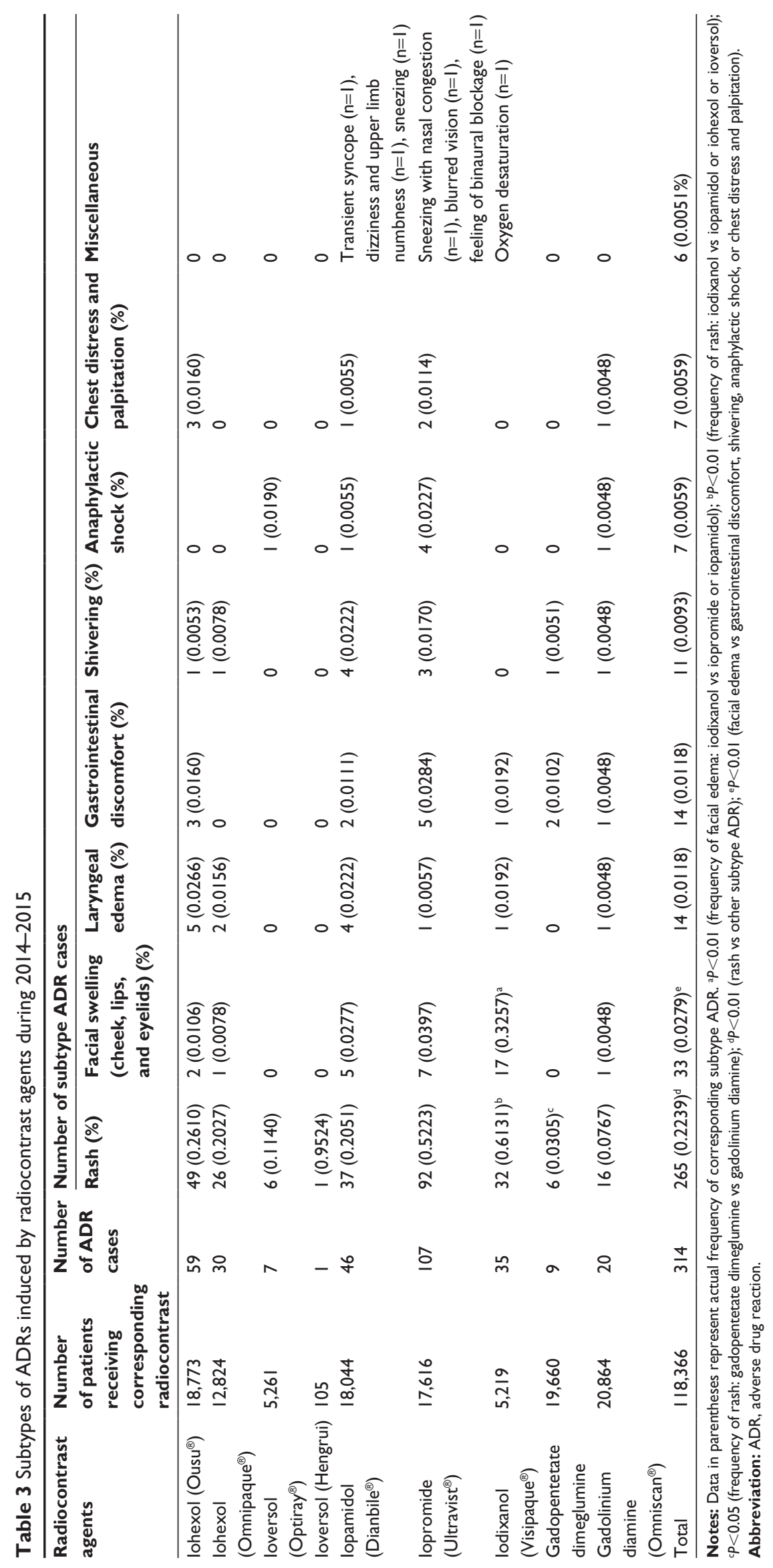


Table 4 Information on allergy history or atopy traits among ADR cases during 20I4-20I5

\begin{tabular}{|c|c|c|}
\hline Radiocontrast agents & $\begin{array}{l}\text { The ratio of ADR cases } \\
\text { with allergy history or } \\
\text { atopy traits to all ADRs } \\
\text { induced by corresponding } \\
\text { radiocontrast agent }\end{array}$ & Details \\
\hline lohexol $\left(\mathrm{Ousu}^{\circledR}\right)$ & $16.95 \%(10 / 59)$ & - Drugs involving allergy: antibacterials $\left(n=50\right.$, ie, penicillins $[n=33]^{a}$, cephalosporins \\
\hline lohexol (Omnipaque $\left.{ }^{\circledR}\right)$ & $23.33 \%(7 / 30)$ & {$[n=7]$, sulfonamides $[n=5]$, metronidazole $[n=1]$, streptomycin $[n=1]$, gentamicin } \\
\hline lopamidol (Dianbile) & $19.57 \%(9 / 46)$ & $[\mathrm{n}=\mathrm{I}]$, an unknown antibiotics $[\mathrm{n}=\mathrm{I}]$, ofloxacin $[\mathrm{n}=\mathrm{I}])$, Shenmai injection \\
\hline lopromide (Ultravist ${ }^{\circledR}$ ) & $24.30 \%(26 / 107)$ & (a traditional Chinese medicine) $(n=I)$, Shuanghuanglian injection $(n=I)$, calcitonin \\
\hline lodixanol (Visipaque $\left.{ }^{\circledR}\right)$ & $20.00 \%(7 / 35)$ & $(n=I)$, procaine $(n=I)$, tetanus antitoxin $(n=I)$, iodine contrast agent $(n=I)$, iodine \\
\hline loversol (Optiray $\left.{ }^{\circledR}\right)$ & $14.29 \%(1 / 7)$ & $(n=I)$, Ultravist $(n=I)$, metamizole sodium $(n=I)$, Contac NT $(n=I)$. \\
\hline loversol (Hengrui) & $100.00 \%(I / I)$ & - Food and other substance: pollen $(n=2)$, mango $(n=I)$, cicadas $(n=I)$, mulberry \\
\hline Gadopentetate dimeglumine & $33.33 \%(3 / 9)$ & $(n=1)$, seafood $(n=4)$, adhesive tape $(n=2)$, dust mite $(n=2)$, paint $(n=2)$. \\
\hline Gadolinium diamine $\left(\mathrm{Omniscan}^{\circledR}\right)$ & $25.00 \%(5 / 20)$ & - Atopy: history of rubella $(n=I)$, history of eczema $(n=I)$, rhinallergosis $(n=I)$. \\
\hline
\end{tabular}

Notes: $\mathrm{P}<0.0 \mathrm{O}$. The ratio of number of $A D R$ cases with allergy history of corresponding category of antibacterials to total number of ADR cases: penicillins (I0.5I\%) vs cephalosporins $(2.23 \%)$ or sulfonamides (1.59\%).

Abbreviation: ADR, adverse drug reaction.

subtype (relative percentage: 84.39 ; actual frequency: $0.2239 \%$ ), followed by facial swelling (cheek, lip, and eyelid) (relative percentage: 10.51; actual frequency: 0.0279\%), laryngeal edema (relative percentage: 4.46 ; actual frequency: $0.0118 \%$ ), gastrointestinal discomfort (relative percentage: 4.46; actual frequency: $0.0118 \%$ ), shivering (relative percentage: 3.50 ; actual frequency: $0.0093 \%$ ), anaphylactic shock (relative percentage: 2.23; actual frequency: $0.0059 \%$ ), and chest distress and palpitation (relative percentage: 2.23 ; actual frequency: $0.0059 \%)$. There was statistically significant difference in frequency of ADR subtype induced by radiocontrast agents (rash $>$ facial swelling [cheek, lip, and eyelid] $>$ gastrointestinal discomfort, shivering, anaphylactic shock, chest distress, and palpitation, $P<0.01$ ). More facial swelling was observed in patients receiving iodixanol compared with iopamidol and iopromide $(P<0.01)$. Higher frequency of rash was observed in patients receiving iodixanol compared with iohexol, iopamidol, or ioversol $(P<0.01)$. Frequency of rash was lower in gadopentetate dimeglumine group compared with gadolinium diamine group $(P<0.05)$.

\section{Allergy history or atopy traits among ADR cases}

Table 4 lists information on allergy history or atopy traits among ADR cases during 2014-2015. Among 314 cases of ADRs induced by radiocontrast agents, 69 patients (21.97\%) had allergy history $(n=66)$ or atopy traits $(n=3)$. ADR cases with allergy history of antibacterials accounted for $83.33 \%$ (50/60) of patients with drug allergy history. The ratio of number of ADR cases with allergy history of penicillins to all ADR cases $(10.51 \%, 33 / 314)$ was higher compared with cephalosporins $(2.229 \%, 7 / 314)$ or sulfonamides $(1.592 \%$,
5/314) $(P<0.01)$. Among 265 cases of rash, 55 patients had allergy history $(20.75 \%, 55 / 265)$. Five patients had allergy history among 14 laryngeal edema patients (35.71\%, 5/14), and 4 patients had allergy history among 21 facial swelling patients $(19.05 \%, 4 / 21)$.

\section{Time to onset of ADRs}

Table 5 lists time to onset of ADRs related with radiocontrast agents during 2014-2015. Patients with allergy history or atopy traits experienced ADRs earlier than those without allergy history or atopy traits during 2014-2015 $(17.19 \pm 43.59 \mathrm{~min}$ vs $85.34 \pm 253.82 \mathrm{~min}, P<0.01)$ and in $2014(20.36 \pm 55.07 \mathrm{~min}$ vs $115.56 \pm 280.20 \mathrm{~min}, P<0.01)$. Time to onset of ADRs was increased in patients receiving iodixanol compared with patients receiving non-iodixanol iodinated radiocontrast agents $(323.77 \pm 386.17 \mathrm{~min}$ vs $42.36 \pm 186.61 \mathrm{~min}, P<0.01)$. A total of $84.08 \%(264 / 314)$ of ADR cases occurred within $30 \mathrm{~min}$. The proportion of delayed ADRs, which occurred over $1 \mathrm{~h}$ after contrast medium injection, was less than that within 5 min $(9.55 \%$ vs $37.26 \%, P<0.01)$. There was no significant difference in the proportion of ADR occurrence within 5 min between 2014 and 2015 (38.89\% vs $35.07 \%, P>0.05)$; however, the proportion of ADR occurred beyond $1 \mathrm{~h}$ decreased from $13.89 \%$ to $3.73 \%$ (2014 vs $2015, P<0.01)$.

\section{Discussion}

The study included 118,208 patients enrolled in various types of enhanced CT or MRI examinations, which could reflect ADR conditions in Chinese population accurately and reliably. Regarding frequency of ADRs, "rare" means the occurrence rate $\geq 0.01 \%$ but $<0.1 \%$ whereas "uncommon" 
Table 5 Time to onset of ADRs related to radiocontrast agents during 20I4-20I5

\begin{tabular}{|c|c|c|c|c|c|c|}
\hline \multirow[t]{2}{*}{ Investigation items } & \multicolumn{2}{|c|}{2014} & \multicolumn{2}{|c|}{2015} & \multicolumn{2}{|c|}{2014 and 2015} \\
\hline & $\mathbf{n}$ & $\begin{array}{l}\text { Time to onset } \\
\text { of ADRs ( } \mathrm{min} \text { ) }\end{array}$ & $\mathbf{n}$ & $\begin{array}{l}\text { Time to onset } \\
\text { of ADRs (min) }\end{array}$ & $\mathbf{n}$ & $\begin{array}{l}\text { Time to onset } \\
\text { of ADRs ( } \mathrm{min} \text { ) }\end{array}$ \\
\hline Patients with allergy history or atopy traits & 42 & $20.36 \pm 55.07^{a}$ & 27 & $|2.08 \pm 8.6|$ & 69 & $17.19 \pm 43.59^{\mathrm{a}}$ \\
\hline Patients without allergy history or atopy traits & 138 & || $5.56 \pm 280.20$ & 107 & $46.73 \pm 210.48$ & 245 & $85.34 \pm 253.82$ \\
\hline lodixanol & 35 & $323.77 \pm 386.17^{b}$ & 0 & & 35 & $323.77 \pm 386.17^{b}$ \\
\hline Non-iodixanol iodinated radiocontrast* & 135 & $40.30 \pm 171.90$ & 115 & & 250 & $42.36 \pm|86.6|$ \\
\hline \multicolumn{7}{|l|}{ Time to onset of ADRs } \\
\hline$\leq 5 \mathrm{~min}$ & \multicolumn{2}{|c|}{$38.89 \%(70 / 180)$} & \multicolumn{2}{|c|}{$35.07 \%(47 / 134)$} & \multicolumn{2}{|c|}{$37.26 \%(117 / 3 \mid 4)$} \\
\hline $5-30 \mathrm{~min}$ & \multicolumn{2}{|c|}{$41.11 \%(74 / 180)$} & \multicolumn{2}{|c|}{$54.48 \%(73 / 134)^{c}$} & \multicolumn{2}{|c|}{$46.82 \%(|47 / 3| 4)$} \\
\hline$>30 \mathrm{~min}$ & \multicolumn{2}{|c|}{$19.44 \%(35 / 180)^{e}$} & \multicolumn{2}{|c|}{$11.19 \%(15 / 134)^{\mathrm{c}, \mathrm{e}}$} & \multicolumn{2}{|c|}{$15.92 \%(50 / 3 \mid 4)^{\mathrm{e}}$} \\
\hline$>\mathrm{lh}$ & \multicolumn{2}{|c|}{$13.89 \%(25 / 180)^{e}$} & \multicolumn{2}{|c|}{$3.73 \%(5 / 134)^{d, e}$} & \multicolumn{2}{|c|}{$9.55 \%(30 / 3 \mid 4)^{\mathrm{e}}$} \\
\hline
\end{tabular}

Notes: *Non-iodixanol iodinated contrast medium included iopamidol, iohexol, iopromide, and ioversol. Student's $t$-test, ${ }^{a} P<0.01$ (patients with allergy history or atopy vs patients without allergy history or atopy), ${ }^{b} P<0.0$ I (iodixanol vs non-iodixanol iodinated contrast medium), ${ }^{c} P<0.05,{ }^{d} P<0.0$ I $\left(2014\right.$ vs 20 I 5 ), ${ }^{e} P<0.01$ ( $>$ I $\mathrm{h} \mathrm{vs} \leq 5$ min or $>30 \mathrm{~min}$ vs $\leq 5 \mathrm{~min})$. Data presented as mean \pm standard deviation unless stated otherwise.

Abbreviation: ADR, adverse drug reaction.

represents the occurrence rate $\geq 0.1 \%$ but $<1 \% .{ }^{8}$ Therefore, moderate or severe ADRs induced by 12 kinds of radiocontrast agents in our study were rare whereas mild ADRs were uncommon and accounted for $83.76 \%$ of ADRs (263/314), suggesting that enhanced CT and MRI examinations are highly safe.

It was anecdotally noted that a substantially lower frequency of ADRs was observed in inpatients compared with outpatients and emergency patients at our institution, indicating radiologists and radiology nurses should maintain close vigilance during CT or MRI examinations for outpatients and emergency patients. Dean et al also observed an unexpected discrepancy between inpatient and outpatient cohorts with respect to frequency of ADRs induced by radiocontrast agents and they supposed the hypotheses that there might be several potential barriers to identifying ADRs within the inpatient workflow or there are more opportunities of identifying skin changes or other manifestations of radiocontrast ADRs for outpatients (eg, the time to change from provided gowns for some MRI examinations to outpatients' own personal clothing). ${ }^{9}$ There seems to be no barrier to identify radiocontrast-induced ADRs among inpatients at our institution. Both inpatients and outpatients are usually required to move to a holding area for at least $30 \mathrm{~min}$ and obvious non-laboratory ADRs induced by radiocontrast agents can be identified and communicated to the radiology team. If inpatients exhibit ADRs induced by radiocontrast agents after going back to their wards, their ward nurses would enter relevant ADR information into the electronic medical recording system (EMRS) and contact radiology nurses who would read the ADR information via EMRS and then record them on specific written sheets. However, ward nurses' ADR reporting and communicating with radiology nurses are voluntary and thus it is possible to omit collecting ADRs induced by radiocontrast agents, although JCI-accredited SAHZU has an excellent safety culture. Moreover, an intensive care unit (ICU) patient receiving contrast medium at radiology department would be sent back to ICU immediately and it is possible to neglect whether an ICU patient in coma is allergic to the contrast medium. Further research is needed to delineate the exact etiology of the difference in ADR reporting between patient setting type.

Li et al reported that female patients had significantly higher incidence rates of ADRs caused by non-ionic iodinated contrast media compared with male patients $(0.40 \%$ vs $0.30 \% ; P<0.01) .{ }^{2}$ Our study also confirmed that sex could affect the development of ADRs associated with enhanced CT examination to a certain degree. The effect of sex on ADR frequency was not observed in patients receiving gadolinium contrast agents, which was not in accordance with previous findings that adverse events were more likely in women. The underlying mechanism is still uncertain.

An interesting phenomenon was observed in our study, that is, patients with positive allergy history or atopy traits experienced ADRs earlier than those who were negative. To our knowledge, this is the first report to reveal the effect of allergy history or atopy traits on time to onset of ADRs induced by radiocontrast agents. The fact that $37.2 \%$ of ADR cases occurred within $5 \mathrm{~min}$ and $84.08 \%$ of ADRs occurred within 30 min indicates that radiologists and nurses should pay special attention to these patients immediately after radiocontrast injection, during and shortly after examinations. Patients should not leave the CT room until $30 \mathrm{~min}$ after the examination. Statistically significant decrease in 
the relative contribution of ADRs occurred 30 min after medium injection, which might facilitate the management of ADRs in patients.

Statistically significant difference in frequencies of ADRs was observed with iodinated contrast agents in both 2014 and $2015(P<0.01)$, indicating these differences among different agents should be considered when selecting a medium and when monitoring patients during and after its use to ensure optimum usage and patient safety. Zhang et al observed that the incidence of acute adverse reactions was higher with Ultravist-370 (iopromide) than with Isovue-370 (iopamidol) $(0.38 \%$ vs $0.24 \%, P<0.001)$, but only for mild ADRs $(0.32 \%$ vs $0.16 \%, P<0.001) .{ }^{10}$ In our study, frequency of ADRs induced by iopromide $(0.8115 \%)$ was higher compared with iopamidol $(0.2639 \%)$, ioversol $(0.1976 \%)$, or iohexol $(0.3072 \%)(P<0.01)$, but this difference was only limited to mild ADRs. Two products of gadopentetate dimeglumine exhibited significant difference in frequencies of ADRs $(0.2670 \%$ vs $0.0218 \%, P<0.05)$, indicating possible difference in quality of pharmaceutical formulations with same generic name. It is noteworthy to further investigate the underlying mechanism.

Since the beginning of 2015, SAHZU has initiated a quality improvement program of reducing ADRs induced by radiocontrast agents. Comprehensive intervention measures were as follows:

1) Physicians are required to pay more attention to clinical indications and contraindications of radiocontrast examinations, assess renal function and obtain informed consent from patients.

2) Radiology nurses are required to strengthen patient education and pre-examination screening of key information (eg, integrity of signed informed consent, allergy history, atopy traits, comorbidities, renal function, and metformin use). Before 2015, radiology nurses directly initiated radiocontrast injection for patients with bronchial asthma or a history of drug anaphylactic shock if these patients' signed informed consent were complete. However, a new process was established to decrease allergy risk among such patients since the beginning of 2015, that is, patients with history of drug anaphylactic shock will be re-informed with potential risk of radiocontrast use by radiology nurses. If the patient declines to receive radiocontrast examination, the examination will be canceled and alternative examination will be considered by the prescribing physician. If the patient insists on receiving radiocontrast examination, radiology nurse will contact the prescribing physician to confirm the order.
For an asthma patient, radiology nurse will re-assess the patient. If the patient has an allergic asthma, radiocontrast examination must be canceled. Patients with bronchial asthma are generally suggested to avoid the radiocontrast examination except when the patient has been received such examination in the past or the prescribing physician insists on the examination. For such cases, after careful consideration, family members of outpatients and doctor of inpatients are required to accompany them during radiocontrast examination.

3) Since the beginning of 2015, radiologists have to order personalized dose of contrast medium based on a dose calculation algorithm that combines multiple factors such as examination machine, scanning technique, examination site and body weight. On the contrary, dealing with CT radiocontrast doses was all a matter of experience before 2015 (eg, 80-100 mL for chest and abdomen examinations, 60-80 mL for head and neck examinations).

4) Emergency management contingency plan was further improved. When ADR occurs, all clinical professionals at the radiology department should perform rapid evaluation, closely monitor vital signs and pulse oxygen saturation, appropriately use the devices and medications to treat the patients, timely request for help, record and follow-up the patients. For patients experiencing severe ADRs, immediate treatment should be performed, and clinicians from the emergency, anesthesia and other related departments should be asked to perform treatments in cooperation. Cardiopulmonary resuscitation should be performed immediately when cardiorespiratory arrest occurs. Basic life and advanced life support should be given in the radiology department. Patients receiving radiocontrast agents should be closely monitored by clinical professionals for at least 30 min after radiocontrast injection. Nurse head would routinely check the implementation of ADRs recording by nurses on duty. All radiocontrast-related ADRs were analyzed monthly and quarterly and discussed at the DTC meeting.

5) Information technology was further applied, that is, intelligent warnings of disobeying contraindications of radiocontrast examination during physician's prescribing via EMR and online ADR report system.

6) Formulary was updated. According to ADR monitoring data in 2014, frequency of moderate/severe ADRs induced by iodixanol was 4.1-5.4 times that of iohexol, iopromide, or iopamidol. Moreover, time to onset of ADRs induced by iodixanol was $\sim 8$ times that of other iodinated radiocontrast agents. After discussion in the 
DTC meeting, SAHZU took a pragmatic intervention (ie, intravenous iodixanol use in radiocontrast examinations was prohibited in 2015).

SAHZU witnessed statistically significant reduction in occurrence rate of ADRs as well as moderate/severe ADRs induced by iodinated contrast agents from 2014 to 2015 $(P<0.01)$, indicating successful quality improvement by multidisciplinary collaboration among physicians, pharmacists, radiologists, nurses at radiology nursing unit, information technology engineers, and quality management experts.

\section{Limitations}

Our study has some limitations. First, although information on allergy history or atopy traits for each patient must be documented in the SAHZU's EMRS, we are unable to collect the information on allergy history or atopy traits of 117,894 patients who did not experience ADRs induced by radiocontrast agents due to inconvenience in accessibility of big data. Therefore, whether patients with allergy history or atopy traits are more susceptible to ADRs induced by radiocontrast agents was not addressed in this study. Nevertheless, we revealed the effect of allergy history or atopy traits on time to onset of ADRs induced by radiocontrast agents. Second, the ADRs collected in this study are not laboratory based, so ADRs such as acute kidney injuries were not our focus. Third, we only focused on the intravenous use of radiocontrast agents in this study. ADRs occurring due to radiocontrast administration via other administration routes are worthy of being investigated in the future.

\section{Conclusion}

Non-laboratory-based moderate/severe ADRs induced by radiocontrast agents are rare whereas mild ones were uncommon and accounted for the majority of ADRs. Efficient quality improvement in decreasing frequency of ADRs induced by contrast has been achieved by multidisciplinary collaboration. Detailed knowledge of ADRs induced by contrast media could help in promoting risk management and patient safety.

\section{Acknowledgments}

This work was supported by Zhejiang Provincial Bureau of Education (application number Y201635841). Also, we would like to thank Professor Xiao-na Dai (Dean of Department of Quality Administration), Mr Guang-wu Li (Inpatient Pharmacy Supervisor), and Wei He (Clinical Pharmacist) for their kind help with medication management and use.

\section{Disclosure}

The authors report no conflicts of interest in this work.

\section{References}

1. ISMP high-alert medications. [webpage on the Internet] Available from: http://www.ismp.org/Tools/highAlertMedicationLists.asp. Accessed January 26, 2017.

2. Li X, Chen J, Zhang L, et al. Clinical observation of the adverse drug reactions caused by non-ionic iodinated contrast media: results from 109,255 cases who underwent enhanced CT examination in Chongqing, China. Br J Radiol. 2015;88(1047):20140491.

3. Prince MR, Zhang H, Zou Z, Staron RB, Brill PW. Incidence of immediate gadolinium contrast media reactions. AJR Am J Roentgenol. 2011;196(2):W138-W143.

4. Brown JR, Solomon RJ, Sarnak MJ, et al. Reducing contrast-induced acute kidney injury using a regional multicenter quality improvement intervention. Circ Cardiovasc Qual Outcomes. 2014;7(5):693-700.

5. Dykes TM, Bhargavan-Chatfield M, Dyer RB. Intravenous contrast extravasation during $\mathrm{CT}$ : a national data registry and practice quality improvement initiative. J Am Coll Radiol. 2015;12(2):183-191.

6. Working group of Chinese Society of Radiology on the safe use of contrast agents. Guideline for iodine contrast agent use. Natl Med J China. 2014;43:3363-3369.

7. Naranjo CA, Busto U, Sellers EM, et al. A method for estimating the probability of adverse drug reactions. Clin Pharmacol Ther. 1981; 30(2):239-245.

8. Eriksson R, Aagaard L, Jensen LJ, et al. Discrepancies in listed adverse drug reactions in pharmaceutical product information supplied by the regulatory authorities in Denmark and the USA. Pharmacol Res Perspect. 2014;2(3):e00038.

9. Dean KE, Starikov A, Giambrone A, Hentel K, Min R, Loftus M. Adverse reactions to intravenous contrast media: an unexpected discrepancy between inpatient and outpatient cohorts. Clin Imaging. 2015; 39(5):863-865.

10. Zhang B, Dong Y, Liang L, et al. The incidence, classification, and management of acute adverse reactions to the low-osmolar iodinated contrast media Isovue and Ultravist in contrast-enhanced computed tomography scanning. Medicine (Baltimore). 2016;95(12):e3170
Therapeutics and Clinical Risk Management

\section{Publish your work in this journal}

Therapeutics and Clinical Risk Management is an international, peerreviewed journal of clinical therapeutics and risk management, focusing on concise rapid reporting of clinical studies in all therapeutic areas outcomes, safety, and programs for the effective, safe, and sustained use of medicines. This journal is indexed on PubMed Central, CAS,

\section{Dovepress}

EMBase, Scopus and the Elsevier Bibliographic databases. The manuscript management system is completely online and includes a very quick and fair peer-review system, which is all easy to use. Visit http://www.dovepress.com/testimonials.php to read real quotes from published authors.

Submit your manuscript here: http://www.dovepress.com/therapeutics-and-clinical-risk-management-journal 